\title{
Retained Gastric Band Tubing Resulting in Large Bowel Obstruction
}

\author{
Christopher Starnes, MD, Sheilendra S. Mehta, MD, Shinil K. Shah, DO, Kulvinder S. Bajwa, MD, \\ Erik B. Wilson, MD, Peter A. Walker, MD \\ Department of Surgery, University of Texas Medical School at Houston, Houston, TX, USA (Drs. Starnes, Mehta, Shah, \\ Bajwa, Wilson, Walker). \\ Michael E. DeBakey Institute for Comparative Cardiovascular Science and Biomedical Devices, Texas A\&M University, \\ College Station, TX, USA (Dr. Shah).
}

\begin{abstract}
Introduction: Complications due to retained gastric band tubing are rarely reported and frequently include disconnections of the tubing requiring operative revision. Obstruction from adjustable gastric band tubing is an infrequently reported event.

Case Description: The patient presented to us 1 month after removal of a presumed isolated infected port performed at an outside facility. The wound was left open, but the intra-abdominal tubing and band were left in place. Abdominal distention, pain, and peritonitis developed, and the patient was taken to the operating room because of concern regarding an acute intra-abdominal process. Intraoperatively, he was noted to have a sigmoid obstruction from the retained gastric band tubing.

Discussion: Although obstruction from adjustable gastric band tubing is infrequently reported in the literature, it poses a potentially devastating complication. We review the relevant literature and potential issues when dealing with band-related tubing.
\end{abstract}

Key Words: Internal hernia, Adjustable gastric band, Bowel obstruction, Port infection.

Citation Starnes C, Mehta SS, Shah SK, Bajwa KS, Wilson EB, Walker PA. Retained gastric band tubing resulting in large bowel obstruction. CRSLS e2014.002591. DOI: 10.4293/CRSLS.2014.002591

Copyright $\odot 2015$ by SLS, Society of Laparoendoscopic Surgeons. This is an open-access article distributed under the terms of the Creative Commons Attribution-Noncommercial-ShareAlike 3.0 Unported license, which permits unrestricted noncommercial use, distribution, and reproduction in any medium, provided the original author and source are credited.

Christopher Starnes and Sheilendra S. Mehta contributed equally to this study.

Address correspondence to: Shinil K. Shah, DO, Department of Surgery, University of Texas Medical School at Houston, 6431 Fannin St, MSB 4.156, Houston, TX 77030, USA. Telephone: (281) 841-6034, E-mail: shinil.k.shah@uth.tmc.edu

\section{INTRODUCTION}

The number of new adjustable gastric bands (AGBs) being placed is decreasing. ${ }^{1}$ Given the hundreds of thousands of bands placed worldwide, ${ }^{1}$ it is imperative, however, for the surgeon to understand the potential complications associated with these devices. We report a relatively uncommon case of retained AGB tubing contributing to a large bowel obstruction.

\section{CASE REPORT}

The patient is a 58-year-old man with a medical history of diabetes, hypertension, myocardial infarction (for which stent placement had been performed), and multiple cerebrovascular accidents, as well as a surgical history of laparoscopic AGB placement about 4 years before presentation. He initially presented to an outside hospital because of an eroded subcutaneous band port. At that facility, he underwent removal of the subcutaneous port. The gastric band and attached tubing were left in place. Upper endoscopy was not performed at that time.

The patient presented to our facility about 1 month later complaining of right lower-quadrant abdominal pain, nausea, anorexia, constipation, and fever present intermittently since removal of the AGB subcutaneous port. The 


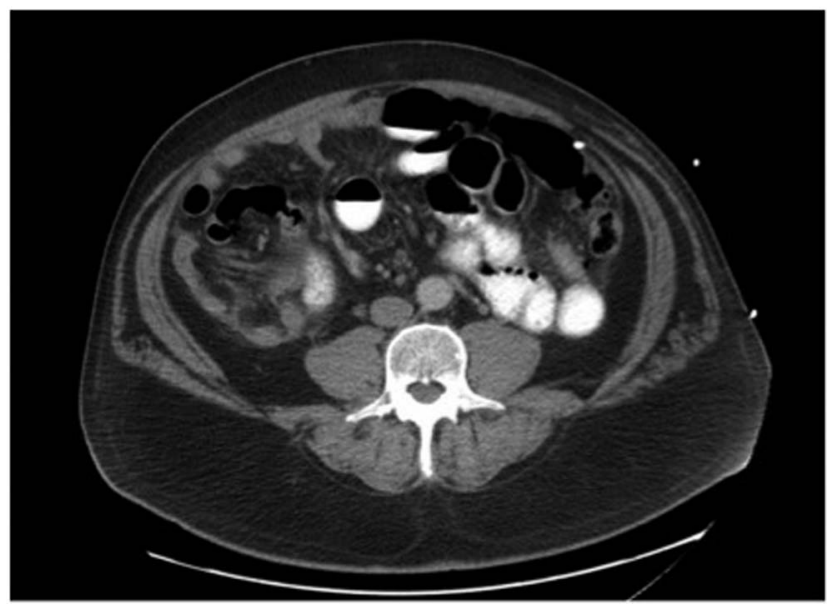

Figure 1. The initial computed tomography scan of the abdomen and pelvis showed dilation of the proximal small bowel with relative decompression of the distal bowel loops, as well as inflammatory changes in the right lower quadrant and ileum.

rest of his medical history was noncontributory. On initial examination, he was afebrile and hypertensive and had a normal heart rate. The physical examination findings were remarkable for right lower-quadrant and suprapubic tenderness. The site of his subcutaneous port had been left open and was without signs of infection. Laboratory study findings were remarkable for a creatinine level of 1.4 $\mathrm{mg} / \mathrm{dL}$, serum glucose level of $166 \mathrm{mg} / \mathrm{dL}$, and white blood cell count of $10600 / \mathrm{mm}^{3}$ ( $78.2 \%$ neutrophils). Computed tomography of the abdomen and pelvis showed mild to moderate small bowel dilation with relative decompression of the distal ileum, with inflammatory wall thickening and associated mesenteric inflammatory changes in the distal ileum (Figure 1).

About 18 hours after admission, peritonitis developed and the patient was taken to the operating room for diagnostic laparoscopy. On entry, the small bowel and large bowel were noted to be significantly dilated; this made examination of the peritoneal cavity difficult. The case was converted to open laparotomy. The sigmoid colon was noted to be redundant and dilated. Further examination showed that the AGB tubing was lying across the proximal and distal sigmoid colon as the tubing traversed toward the right lower quadrant and pelvis (Figure 2). The tubing had become a fixed structure, causing an obstruction of the sigmoid colon. The tubing was mobilized and removed, releasing the sigmoid colon obstruction. The band was then removed from around the stomach. There was no evidence of band erosion. The pelvis and right lower quadrant were evaluated to determine the point of fixa-

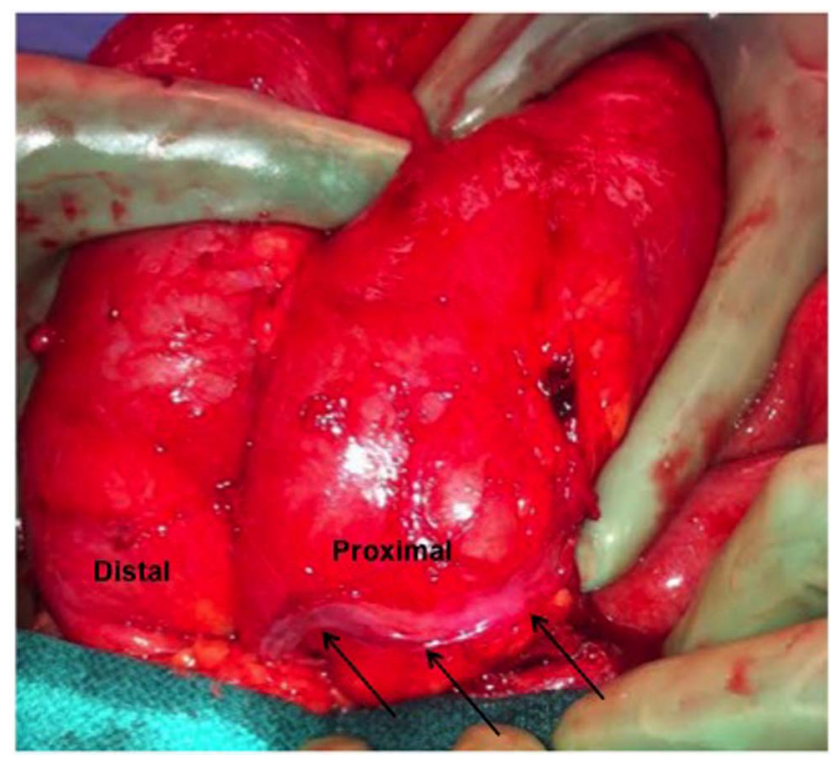

Figure 2. Intraoperative photograph showing where the gastric band tubing had created a partial obstruction of the sigmoid colon (arrows). The sigmoid colon was noted to be redundant, and a serosal indentation where the band tubing initially traversed was noted on the proximal sigmoid colon. The band continued somewhat inferiorly to pass along the distal sigmoid colon, creating a closed-loop obstruction of the sigmoid colon.

tion. The small bowel was examined; several loops of distal ileum were matted together. These were gently dissected free. Fibrinous exudate without gross purulence was seen, and the appendix was noted to be indurated and erythematous. An appendectomy was subsequently performed. Although we had planned to perform intraoperative endoscopy, given the operative findings, as well as the absence of intraoperative evidence of gastric band erosion, the procedure was deferred.

The patient's postoperative course was complicated by acute kidney injury, which resolved without need for hemodialysis. He was discharged on postoperative day 6 . He has had no other adverse events postoperatively. Surgical pathologic analysis showed fibrous obliteration of the appendix. Review of the initial computed tomography scan showed a dilated redundant sigmoid colon and relative decompression of the rectum. The AGB tubing was noted to be coursing in the region of the sigmoid colon (Figure 3).

\section{DISCUSSION}

Commonly reported postoperative issues related to AGBs include slips, erosion, and inadequate weight loss. ${ }^{2,3}$ Most 

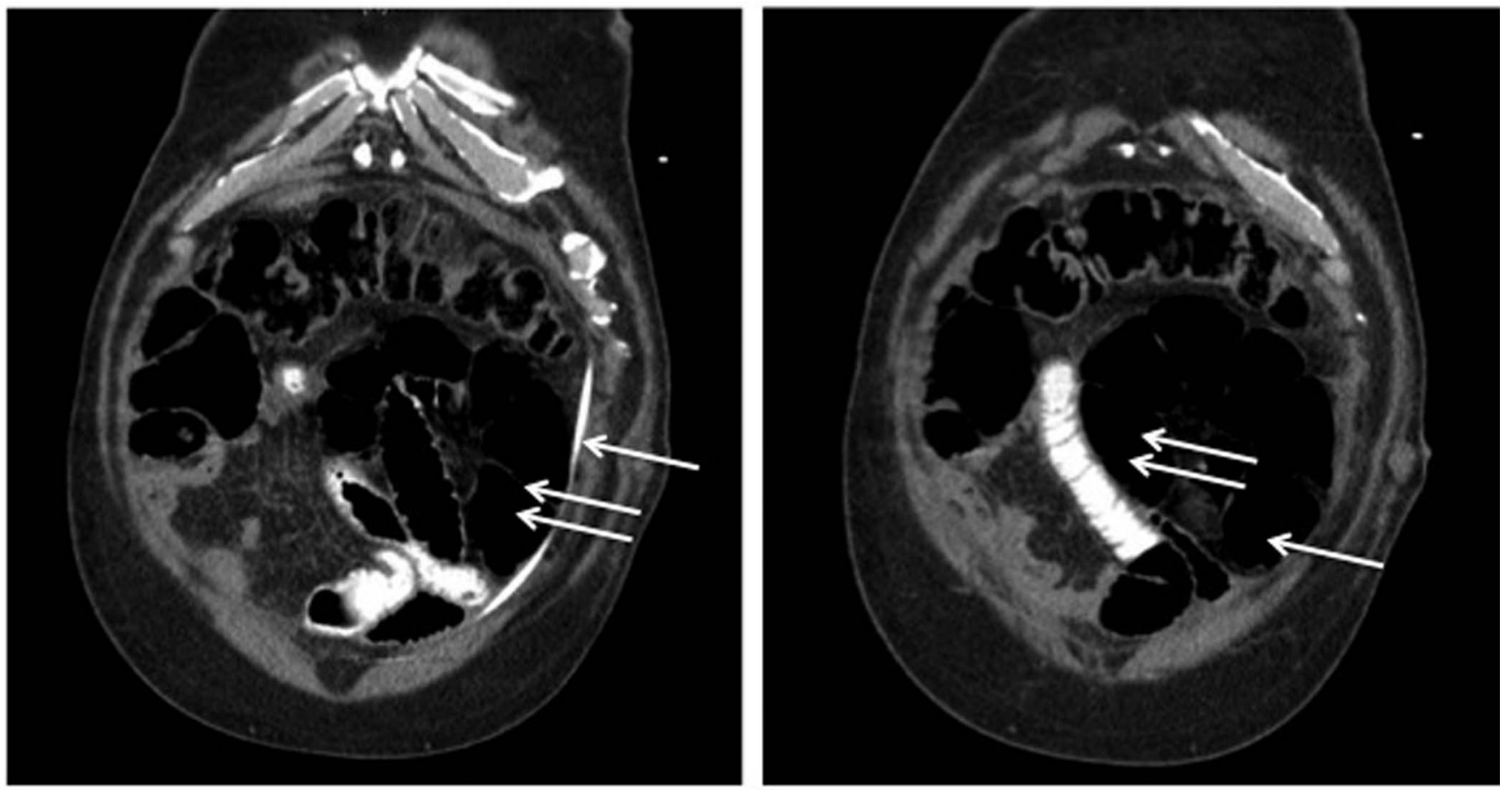

Figure 3. Re-evaluation of the initial computed tomography scan showed the gastric band coursing near a dilated sigmoid colon. On the left image, the single arrow indicates the gastric band tubing and the double arrows indicate the dilated sigmoid colon. On the right image, the single arrow and double arrows indicate the proximal sigmoid colon and distal sigmoid colon, respectively. The rectum was noted to be relatively decompressed (not shown).

surgeons who place AGBs leave laxity in the intraperitoneal tubing of the gastric band, and the tubing is thought of as relatively innocuous. However, as alluded to in the case presented, as well as in the literature referenced, the AGB tubing may be a source of morbidity.

Complications due to AGB-associated tubing are rarely reported in the literature. ${ }^{4-17}$ Much of what is reported in the literature regarding complications related to AGB tubing includes disconnections requiring operative revision. ${ }^{2,3,18,19}$ Other reported complications include bowel obstruction $^{4,6-14}$ and erosion of the tubing into adjacent structures. ${ }^{15,20-28}$ Internal hernias due to AGB tubing have been reported rarely.5,16,17,29,30 In total, fewer than 25 cases of internal hernias or bowel obstructions (or both) due to AGB tubing have been reported. To our knowledge, this is one of the first reported cases of sigmoid colon involvement by AGB tubing. The common theme is that tubing serves as a fixed object between the stomach and the anterior abdominal wall. If there are multiple adhesions along the band and peritoneum, a window for herniation exists. This condition is very similar to an omental adhesion to the abdominal wall creating an in- traperitoneal defect through which viscera can become incarcerated and even strangulated.

In the case presented, relative fixation of the distal tubing in the right lower quadrant as it coursed over the sigmoid colon was initially thought to be due to potential appendicitis. However, the inflammatory changes appeared to be due to band tubing given the absence of evidence of appendicitis on the final surgical pathologic analysis.

Isolated port-site infections should prompt evaluation for gastric band erosion, generally by intraoperative or preoperative endoscopy. ${ }^{31}$ In the reported case, endoscopy was not performed at the time of removal of the infected subcutaneous port. If erosion is ruled out, salvage of the band is possible via removal of the port and replacement at a future date. If retained tubing is left in place, a high index of suspicion is necessary when patients represent with atypical gastrointestinal symptoms. Factors contributing to tubing-related internal hernias or obstruction may include the length of the tubing, lack of omental separation of the tubing and underlying viscera, and previous surgery with intraperitoneal adhesions that may act as 
fixation points. ${ }^{5}$ Reported strategies to prevent these issues include decreasing the length of the tubing, performing anterior abdominal wall or falciform ligament fixation of the tubing, and placing excess tubing (especially when tubing is disconnected as in the case of port-only removal) in the right upper quadrant. 5,17

\section{CONCLUSIONS}

Although internal hernia and obstruction from the intraperitoneal tubing of AGBs are infrequently reported, they are potentially catastrophic complications. Surgeons should have a high index of suspicion when treating patients with abdominal pain in the setting of normal AGB-related imaging, as well as patients with port infections without evidence of band erosion. Plication of excess AGB tubing to the anterior abdominal wall in conjunction with shortening of excess tube length provides a viable option for reducing the incidence of possible tube-related complications.

\section{References:}

1. Buchwald H, Oien DM. Metabolic/bariatric surgery worldwide 2011. Obes Surg. 2013;23:427-436.

2. Sarker S, Herold K, Creech S, Shayani V. Early and late complications following laparoscopic adjustable gastric banding. Am Surg. 2004;70:146-149, discussion 149-150.

3. Peterli R, Donadini A, Peters T, Ackermann C, Tondelli P. Re-operations following laparoscopic adjustable gastric banding. Obes Surg. 2002;12:851-856.

4. Oppliger F, Wiedmaier G, Leon J. Acute small bowel obstruction due to the connecting tube of a gastric band. Surg Obes Relat Dis. 2014;10:e77-e79.

5. Hamed OH, Simpson L, Lomenzo E, Kligman MD. Internal hernia due to adjustable gastric band tubing: review of the literature and illustrative case video. Surg Endosc. 2013;27:43784382.

6. Agahi A, Harle R. A serious but rare complication of laparoscopic adjustable gastric banding: bowel obstruction due to caecal volvulus. Obes Surg. 2009;19:1197-1200.

7. Zappa MA, Lattuada E, Mozzi E, et al. An unusual complication of gastric banding: recurrent small bowel obstruction caused by the connecting tube. Obes Surg. 2006;16:939-941.

8. Shipkov CD, Uchikov AP, Uchikova EH. Small bowel obstruction by the silicone tube of the gastric band. Obes Surg. 2004;14:1280-1282.

9. Petridis C, Neofytou K, Petrou A, Georgiou C. Acute appendicitis as a rare complication of gastric band. J Surg Case Rep. 2013;2013.
10. Hashemzadeh M, Karamirad M, Zahedi-Shoolami L. Laparoscopic adjustable gastric banding connecting tube causing small bowel obstruction and perforation. Case Rep Surg. 2013;2013: 296037.

11. van de Water W, Vogelaar FJ, Willems JM. An unusual complication 4 years after laparoscopic adjustable banding: jejunal obstruction due to the connecting tube. Obes Surg. 2011; 21:131-133.

12. Strobos E, Antanavicius G, Josloff R. Unusual complication: small bowel obstruction caused by tubing of gastric band. Surg Obes Relat Dis. 2009;5:637-640.

13. Chittleborough TJ, Lau SY, Campbell I. Small bowel infarction after herniation through gastric band tubing loop. Surg Obes Relat Dis. 2012;8:809-810.

14. DeNino WF, Forgione PM. Small bowel obstruction from small bowel volvulus and gram-positive peritonitis in laparoscopic adjustable gastric banding. Surg Obes Relat Dis. 2010;6: 211-212.

15. Tan LB, So JB, Shabbir A. Connection tubing causing small bowel obstruction and colonic erosion as a rare complication after laparoscopic gastric banding: a case report. J Med Case Rep. 2012;6:9.

16. Ng MK, Thompson G. Laparoscopic adjustable gastric band tubing: unusual cause of an internal hernia. Surg Obes Relat Dis. 2009;5:517-518.

17. Mills JK, Zakon J, Hung Nguyen M. Images for surgeons. Strangulation of the small bowel mesentery and internal hernia due to the connecting tube of a gastric band. ANZ J Surg. 2008;78:1128-1129.

18. Himpens J, Cadiere GB, Bazi M, Vouche M, Cadiere B, Dapri G. Long-term outcomes of laparoscopic adjustable gastric banding. Arch Surg. 2011;146:802-807.

19. Phillips E, Ponce J, Cunneen SA, et al. Safety and effectiveness of Realize adjustable gastric band: 3-year prospective study in the United States. Surg Obes Relat Dis. 2009;5:588-597.

20. Cintolo JA, Levine MS, Huang S, Dumon K. Intraluminal erosion of laparoscopic gastric band tubing into duodenum with recurrent port-site infections. J Laparoendosc Adv Surg Tech A. 2012;22:591-594.

21. Tekin A. Migration of the connecting tube into small bowel after adjustable gastric banding. Obes Surg. 2010;20:526-529.

22. Navarra G, Musolino C, Centorrino T, De Marco ML, Sarra G, Curro G. Perforation of an adjustable gastric banding connecting tube into distal transverse colon with intra-luminal migration. Obes Surg. 2009;19:125-127.

23. Hartmann J, Scharfenberg M, Paul M, Ablassmaier B. Intracolonic penetration of the laparoscopic adjustable gastric banding tube. Obes Surg. 2006;16:203-205. 
24. Sneijder R, Cense HA, Hunfeld M, Breederveld RS. A rare complication after laparoscopic gastric banding: connectingtube penetration into the hilus of the kidney. Obes Surg. 2009; 19:531-533.

25. Zengin K, Sen B, Ozben V, Taskin M. Detachment of the connecting tube from the port and migration into jejunal wall. Obes Surg. 2006;16:206-207.

26. Iqbal M, Manjunath S, Seenath M, Khan A. Massive upper gastrointestinal hemorrhage: an unusual presentation after laparoscopic adjustable gastric banding due to erosion into the celiac axis. Obes Surg. 2008;18:759-760.

27. Elakkary E, El Essawy D, Gazayerli MM. Enterocutaneous fistula: a rare complication of laparoscopic adjustable gastric banding. Obes Surg. 2005;15:897-900.
28. Pfeiffer JD, Grant J, Lutfi RE. Transanal protrusion of gastric band tubing: a rare complication of laparoscopic adjustable gastric banding. Surg Obes Relat Dis. 2013;9:e23-e24.

29. Keane T, Margulis AR, Dakin GF, Pomp A. Imaging of patients after the Lap-Band System application. Abdom Imaging. 2012;37:690-696.

30. Daetwiler S, Adamina M, Schob O. Intractable abdominal pain following laparoscopic adjustable gastric banding. Obes Surg. 2005;15:1341-1343.

31. Kohn GP, Hansen CA, Gilhome RW, McHenry RC, Spilias DC, Hensman C. Laparoscopic management of gastric band erosions: a 10-year series of 49 cases. Surg Endosc. 2012;26:541545. 